\title{
REMOÇÃO DE FERRO DA SOLUÇÃO SINTÉTICA DE MÍNERIO LIMONÍTICO DE NÍQUEL UTILIZANDO EXTRAÇÃO POR SOLVENTES*
}

\author{
Paula Aliprandini ${ }^{1}$ \\ Mónica Maria Jiménez Correa² \\ Jorge Alberto Soares Tenório ${ }^{3}$ \\ Denise Crocce Romano Espinosa ${ }^{4}$
}

\section{Resumo}

Os minérios limoníticos de níquel apresentam na sua composição metais como ferro, níquel e cobalto, sendo o primeiro o elemento majoritário. A presença majoritária de ferro prejudica a separação dos demais metais que possuem interesse comercial. Para remoção do ferro, técnicas hidrometalúrgicas são usadas. A extração por solventes é uma técnica com foco na separação seletiva de metais. Neste trabalho, foi preparada uma solução sintética baseada nas concentrações dos metais presentes na lixiviação de um minério limonítico de níquel. A solução foi colocada em contato com o extratante Cyanex 272. Este extratante é conhecido pela sua seletividade ao ferro em valores de $\mathrm{pH}$ próximos a 2,0. As amostras foram analisadas utilizando a técnica de Espectroscopia de Fluorescência de Raios X por Energia Dispersiva (EDX). Após concluídos os ensaios foi encontrado que durante a extração do ferro, o cobalto foi coextraído junto, mesmo em pHs entre 0,5 e 2,0, indicando que a presença do ferro altera o comportamento do cobalto.

Palavras-chave: Minério limonítico de níquel; Extração por solventes; Extração de ferro; Coextração de cobalto

\section{IRON REMOVAL FROM SYNTHETIC NICKEL LIMONITE ORE SOLUTION USING SOLVENT EXTRACTION}

\section{Abstract}

Nickel limonite ore is composed for metals as iron, nickel and cobalt. Iron is the major element in the ore. The iron presence affects the recovery of other metals with commercial interesting. Hydrometallurgical techniques are used to remove iron from aqueous solutions. Solvent extraction is a technique used for selective separation of metals. In this study, a synthetic solution was prepared using the concentration of metals from a sulfuric liquor obtained in leaching process of a nickel limonite ore. The solution was mixed with the extractant Cyanex 272, since, at $\mathrm{pH} 2.0$, iron could be selectively extracted using this reagent. The samples were analyzed by Energydispersive X-ray spectroscopy (EDX). After the test it was found that during extraction of iron, cobalt was co-extracted, between $\mathrm{pH}$ range 0.5 and 2.0. Thus, the presence of iron in solutions affects the cobalt behavior.

Keywords: Nickel limonite ore; Solvent extraction; Iron extraction; Cobalt coextraction.

1 Engenheira de Materiais, mestranda do Departamento de Engenharia Química, Escola Politécnica da Universidade de São Paulo, São Paulo, São Paulo, Brasil.

2 Engenheira Química, mestre em ciências, doutoranda do Departamento de Engenharia Química, Escola Politécnica da Universidade de São Paulo, São Paulo, São Paulo, Brasil.

3 Engenheiro Metalurgista, doutor em engenharia metalúrgica, professor titular, Departamento de Engenharia Química, Escola Politécnica da Universidade de São Paulo, São Paulo, São Paulo, Brasil.

4 Engenheira metalurgista, doutora em engenharia metalúrgica, professora associada, Departamento de Engenharia Química, Escola Politécnica da Universidade de São Paulo, São Paulo, São Paulo, Brasil. 


\section{INTRODUÇÃO}

Característico de regiões tropicais e subtropicais, o minério limonítico de níquel é composto pela mistura heterogênea de metais [1][2][3][4]. Dentre os metais presentes estão o ferro, o níquel e o cobalto. O primeiro se destaca pela presença majoritária no minério e os dois últimos pelo foco comercial [2][3].

O beneficiamento de minérios, incluindo o minério limonítico de níquel, é feito através do processamento hidrometalúrgico [5]. O fluxograma simplificado do processo de hidrometalurgia é mostrado na Figura 1 [2][6]. O minério em questão passa pelo processo de dissolução em uma solução aquosa contendo ácido sulfúrico $\left(\mathrm{H}_{2} \mathrm{SO}_{4}\right)$ e posteriormente esse licor é tratado por uma técnica de recuperação de metais.

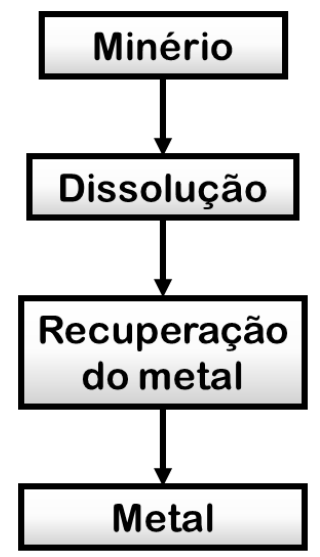

Figura 1: Fluxograma simplificado do processo hidrometalúrgico para o beneficiamento de minério limonítico de níquel [2][6].

A técnica de extração por solventes é utilizada na separação e purificação de soluções contendo diferentes metais, inclusive no beneficiamento do minério limonítico de níquel [3][7][8]. Trata-se de colocar em contato dois líquidos imiscíveis através de agitação, sendo uma fase aquosa e a outra fase orgânica [8][9][10]. Nesse processo, o metal presente na fase aquosa é transferido para a fase orgânica através da interação do extratante com o íon metálico conforme a Equação 1 [11], sendo $\mathrm{M}^{\mathrm{n}+} \mathrm{o}$ íon metálico de interesse e $\mathrm{R}$ a molécula do extratante.

$$
\mathrm{M}^{\mathrm{n}+}+\mathrm{nRH} \leftrightarrow \mathrm{MR}_{\mathrm{n}}+\mathrm{nH}^{+}
$$

Equação 1

O processo de extração por solventes apresenta relação direta com o pH da solução, já que durante a transferência do íon metálico entre as fases há liberação de íons $\mathrm{H}^{+}$ pela fase orgânica [2][11][12].

A escolha do extratante é baseada na seletividade deste frente ao metal de interesse na solução. Para o lixiviado de minério limonítico de níquel, o extratante comercial Cyanex 272 é utilizado na recuperação de metais. $A$

Figura 2 mostra o composto ativo do Cyanex 272 bis(2,4,4-trimetilpentil)fosfínico [13]. 


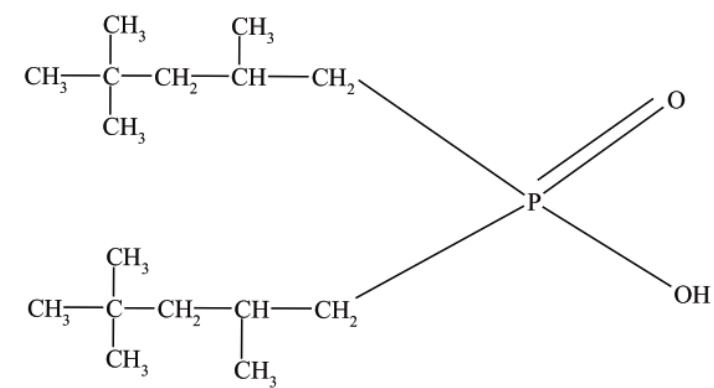

Figura 2: Composto bis(2,4,4-trimetilpentil)fosfínico presente no extratante Cyanex 272 [13].

O Cyanex 272 apresenta a seguinte ordem de seletividade para os metais [14][15]:

$$
\mathrm{Fe}^{+3}>\mathrm{Zn}>\mathrm{Cu}>\mathrm{Co}>\mathrm{Mg}>\mathrm{Ca}>\mathrm{Ni}
$$

Tipicamente o pH de extração dos metais em meio sulfúrico com Cyanex 272 é entre 2 e 3 para o ferro, entre 5 e 6 para o cobalto e entre 7 e 8 para o níquel [16].

Para quantificar o processo de extração por solventes algumas relações podem ser feitas. Uma delas é o coeficiente de distribuição $D$. Este parâmetro prevê a distribuição do íon entre a fase orgânica e a fase aquosa. $D$ é a razão entre a concentração do íon metálico na fase orgânica $[A]$ o e a concentração desse mesmo íon metálico na fase aquosa [A]a quando o sistema está em equilíbrio (Equação 2) [2][7][9][10][17].

$$
D=\frac{[A] o}{[A] a}
$$

\section{Equação 2}

A partir disso é possível inferir que quanto maior o valor de $D$ maior será a extração para a fase orgânica.

Devido à concentração de ferro, o beneficiamento do licor limonítico de níquel para a separação seletiva dos metais é prejudicada [18]. Portanto, para que ocorra a recuperação dos metais como cobalto e níquel, primeiramente é necessário remover o ferro da solução.

Este trabalho tem como objetivo avaliar a remoção do ferro utilizando extração por solventes a partir da variação de pH da solução.

\section{MATERIAIS E MÉTODOS}

A solução utilizada para este estudo foi baseada nos metais e suas respectivas concentrações contidas no licor de lixiviação do minério limonítico de níquel. A composição da solução sintética está descrita na Tabela 1 e foi ajustada para pH 0,5. 
Tabela 1: Composição da solução sintética baseada no licor lixiviado de minério limonítico de níquel.

\begin{tabular}{|c|c|c|}
\hline Metal & Sulfato & $\begin{array}{c}\text { Concentração do } \\
\text { metal }(\mathrm{g} / \mathrm{L})\end{array}$ \\
\hline $\mathrm{Al}$ & $\mathrm{Al}_{2}\left(\mathrm{SO}_{4}\right)_{3} \cdot 14-18 \mathrm{H}_{2} \mathrm{O}$ & 4,57 \\
\hline $\mathrm{Co}$ & $\mathrm{CoSO}_{4} \cdot 7 \mathrm{H}_{2} \mathrm{O}$ & 0,08 \\
\hline $\mathrm{Cu}$ & $\mathrm{CuSO}_{4} \cdot 5 \mathrm{H}_{2} \mathrm{O}$ & 0,14 \\
\hline $\mathrm{Fe}$ & $\mathrm{Fe}_{2}\left(\mathrm{SO}_{4}\right)_{3} \cdot \mathrm{x} \mathrm{H}_{2} \mathrm{O}$ & 21,40 \\
\hline $\mathrm{Mg}$ & $\mathrm{MgSO}_{4} \cdot 7 \mathrm{H}_{2} \mathrm{O}$ & 8,09 \\
\hline $\mathrm{Mn}$ & $\mathrm{MnSO}_{4} \cdot \mathrm{H}_{2} \mathrm{O}$ & 0,37 \\
\hline $\mathrm{Ni}$ & $\mathrm{NiSO}_{4} \cdot 6 \mathrm{H}_{2} \mathrm{O}$ & 2,52 \\
\hline $\mathrm{Zn}$ & $\mathrm{ZnSO}_{4} \cdot 7 \mathrm{H}_{2} \mathrm{O}$ & 0,04 \\
\hline $\mathrm{Cr}$ & $\mathrm{Cr}_{2}\left(\mathrm{SO}_{4}\right)_{3} \cdot \mathrm{x} \mathrm{H}_{2} \mathrm{O}$ & 0,22 \\
\hline
\end{tabular}

A solução orgânica utilizada foi composta pelo extratante Cyanex 272 diluído $20 \%$ em querosene.

A solução sintética foi primeiramente ajustada ao $\mathrm{pH}$ de trabalho com a adição de hidróxido de sódio ( $\mathrm{NaOH}$ ). Os valores de $\mathrm{pH}$ estudados foram: 0,5; 1,0; 1,5 e 2,0. Após o ajuste do pH a solução orgânica foi posta em contato com a solução sintética por agitação constante durante 10 minutos. Durante este período o pH controlado pela adição da base. Ao fim do tempo de contato as duas fases foram vertidas num funil de separação e aguardado até a completa separação das fases. A fase aquosa foi então filtrada em papel de filtro 1PS a fim de garantir que a fase orgânica permanecesse no papel de filtro.

Soluções monoelementares dos metais ferro e cobalto também foram produzidas nas concentrações da Tabela 1. A avaliação de extração por solventes ocorreu utilizando as mesmas condições de trabalho da extração por solventes da solução multielementar.

A técnica de Espectroscopia de Fluorescência de Raios X por Energia Dispersiva (EDX) foi usada para determinar a concentração dos metais nas fases aquosas após o ensaio de extração por solventes.

\section{RESULTADOS E DISCUSSÃO}

O extratante Cyanex 272 é seletivo para o ferro, principalmente em valores de $\mathrm{pH}$ abaixo de 3,0 [19]. Sendo assim é esperado que a extração desse metal para a fase orgânica seja significativa em condições ácidas de experimento.

Os resultados da distribuição dos metais entre as fases aquosa e orgânica mostram que não houve extração para os metais magnésio, alumínio, manganês, níquel, cobre, cromo e zinco (Tabela 2).

Tabela 2: Coeficiente de distribuição $(D)$ dos metais na fase orgânica após a extração por solventes nos respectivos valores de $\mathrm{pH}$ ensaiados.

\begin{tabular}{|r|c|c|c|c|c|c|c|c|c|}
\hline & \multicolumn{10}{|c|}{ Coeficiente de distribuição $(D)$} \\
\hline $\mathrm{pH}$ & $\mathrm{Mg}$ & $\mathrm{Al}$ & $\mathrm{Cr}$ & $\mathrm{Mn}$ & $\mathrm{Fe}$ & $\mathrm{Ni}$ & $\mathrm{Cu}$ & $\mathrm{Zn}$ & $\mathrm{Co}$ \\
\hline 0,5 & 0,00 & 0,00 & 0,07 & 0,06 & 0,11 & 0,05 & 0,06 & 0,06 & 0,12 \\
\hline 1,0 & 0,00 & 0,00 & 0,03 & 0,00 & 0,16 & 0,00 & 0,00 & 0,00 & 0,13 \\
\hline 1,5 & 0,00 & 0,00 & 0,03 & 0,00 & 0,33 & 0,00 & 0,00 & 0,00 & 0,24 \\
\hline 2,0 & 0,00 & 0,00 & 0,03 & 0,00 & 1,82 & 0,00 & 0,00 & 0,00 & 0,94 \\
\hline
\end{tabular}


Como esperado houve crescente extração do ferro ao longo dos valores de $\mathrm{pH}$ analisados. Esse comportamento mostra que há dependência da quantidade de extração e o pH de trabalho, ou seja, tornar o meio alcalino favorece a extração do metal [16].

No entanto o comportamento do cobalto foi diferente do esperado para os valores de pH estudados. $\mathrm{O}$ pH de extração do cobalto para o Cyanex 272 é, teoricamente, acima de 5,0. Porém, como pode ser observado na Figura 3, o metal segue a tendência de extração do ferro, sugerindo que houve coextração.

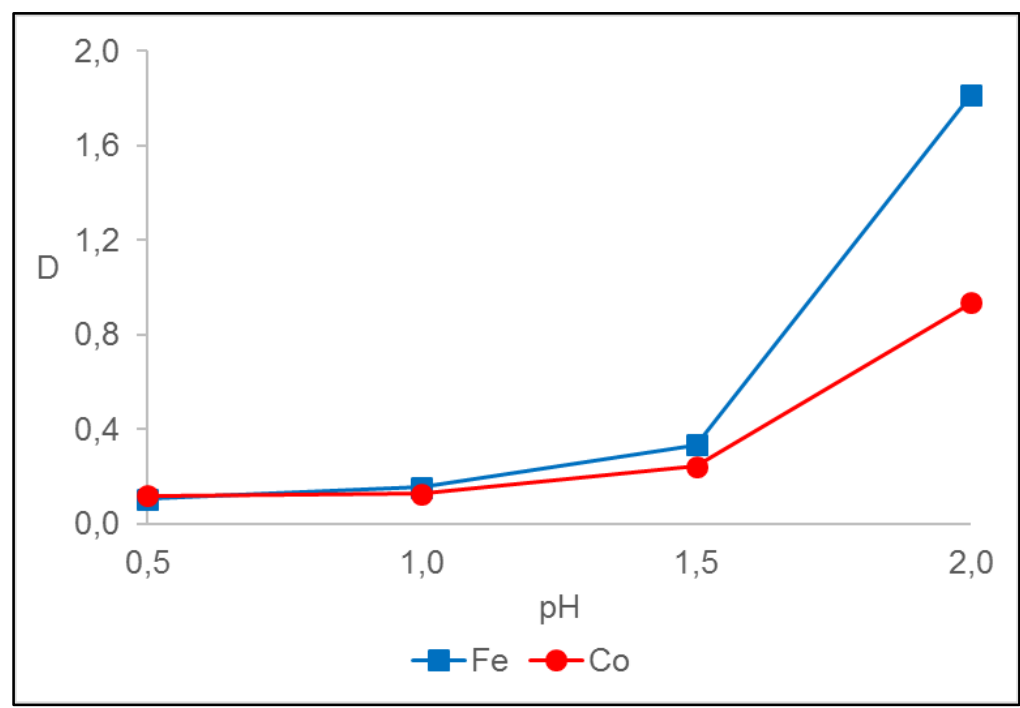

Figura 3: Coeficiente de distribuição $D$ para os metais ferro e cobalto na extração por solventes com Cyanex 272 em função do pH para o licor sintético de minério limonítico de níquel.

A fim de comparar o comportamento dos metais ferro e cobalto na presença de outros metais e em soluções monoelementares, foi realizado o ensaio de extração por solventes para cada solução contendo exclusivamente um dos metais. Foram mantidas as mesmas condições do ensaio da solução multielementar.

A Tabela 3 mostra os valores de $D$ para o ferro nos valores de $\mathrm{pH}$ estudados. É possível ver que o comportamento do ferro independe da presença de outros metais em solução (Figura 4). Esse comportamento já foi verificado por outros autores, confirmando que para valores de $\mathrm{pH}$ em torno de 2,0, o ferro manteve o comportamento de extração independente da presença de outros metais em solução [20].

Tabela 3: Valores comparativos dos coeficientes de distribuição para o ferro em soluções multielementar e monoelementar no intervalo de $\mathrm{pH}$ 0,5-2,0.

Coeficiente de distribuição $(D)$

\begin{tabular}{|c|c|c|}
\hline $\mathrm{pH}$ & $\begin{array}{c}\mathrm{Fe} \\
\text { multielementar }\end{array}$ & $\begin{array}{c}\mathrm{Fe} \\
\text { monoelementar }\end{array}$ \\
\hline 0,5 & 0,11 & 0,07 \\
\hline 1,0 & 0,16 & 0,14 \\
\hline 1,5 & 0,33 & 0,37 \\
\hline 2,0 & 1,82 & 1,99 \\
\hline
\end{tabular}




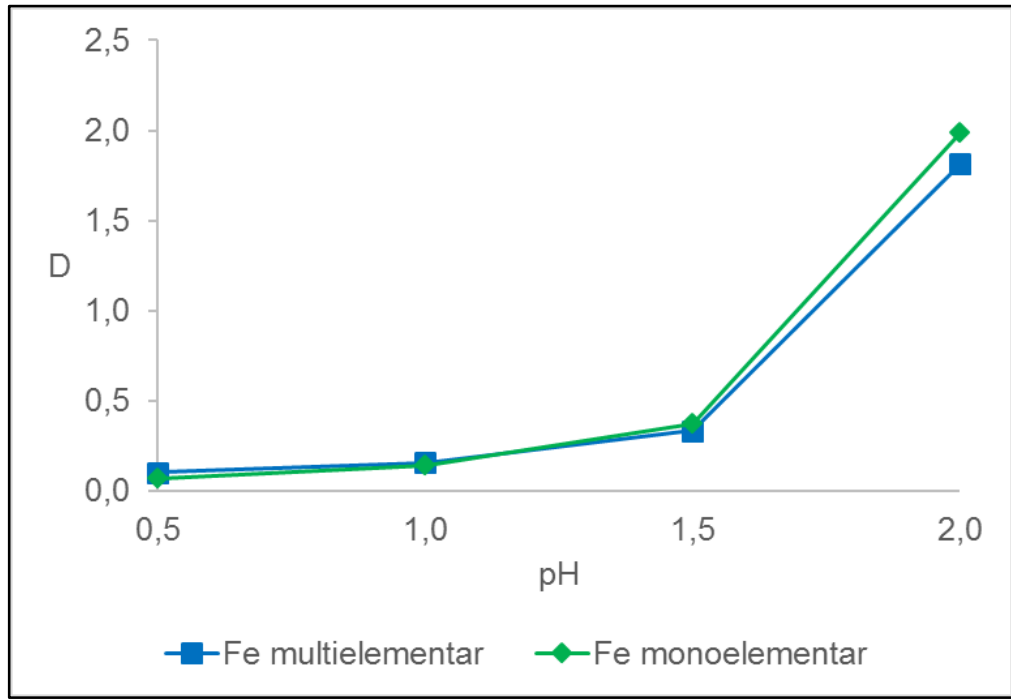

Figura 4: Coeficiente de distribuição do metal ferro para as soluções multielementar e monoelementar nos valores de $\mathrm{pH}$ estudados.

No entanto, a Tabela 4 e a Figura 5 mostram o comparativo entre as extrações para as soluções contendo os outros metais (multielementar) e uma solução contendo apenas o cobalto em solução (monoelementar). É possível observar que há diferença de extração entre as duas soluções. Ao contrário da solução multielementar, a solução monoelementar apresenta o $D$ próximo a zero, indicando que a íon não foi extraído para a solução orgânica.

Tabela 4: Valores comparativos dos coeficientes de distribuição para o cobalto em soluções multielementar e monoelementar no intervalo de $\mathrm{pH}$ 0,5-2,0.

\begin{tabular}{|c|c|c|}
\hline & \multicolumn{2}{|c|}{ Coeficiente de distribuição $(D)$} \\
\hline pH & $\begin{array}{c}\text { Co } \\
\text { multielementar }\end{array}$ & $\begin{array}{c}\text { Co } \\
\text { monoelementar }\end{array}$ \\
\hline 0,5 & 0,12 & 0,03 \\
\hline 1,0 & 0,13 & 0,02 \\
\hline 1,5 & 0,24 & 0,05 \\
\hline 2,0 & 0,94 & 0,05 \\
\hline
\end{tabular}




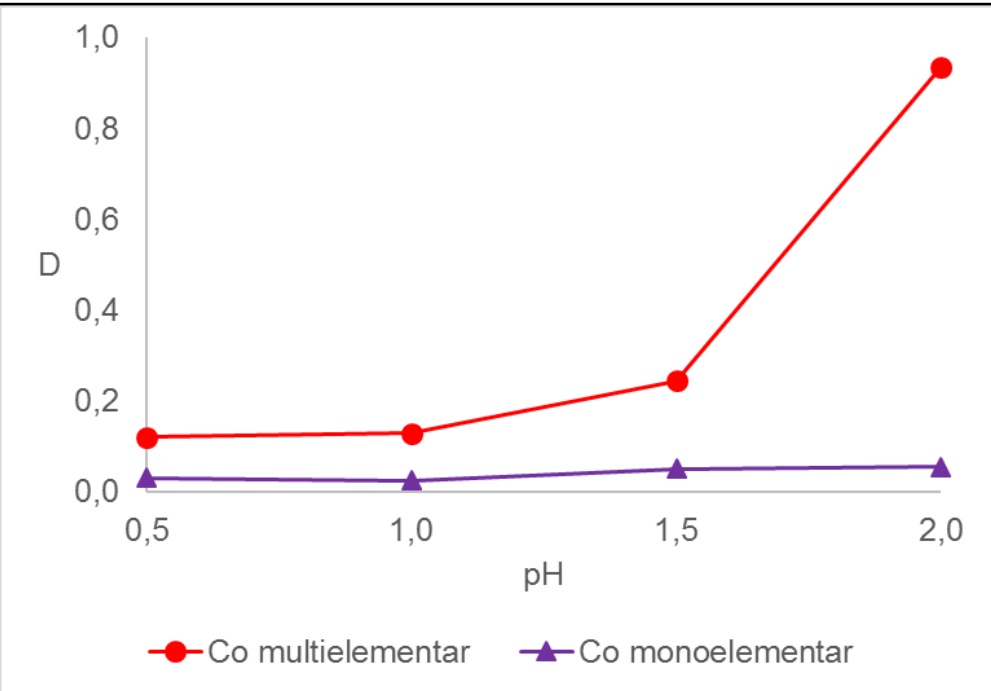

Figura 5: Coeficiente de distribuição do metal cobalto para as soluções multielementar e monoelementar nos valores de $\mathrm{pH}$ estudados.

Estudos comparativos já realizados para a separação de metais através de extração por solventes em soluções multielementares e monoelementares mostrou as variações dos valores de extração devido a interação dos metais.

Doyle et. al. [20] estudou a diferença de extração entre soluções de metais individuais e na presença do íon ferro. As conclusões foram que a presença do ferro em solução afetou a extração dos demais metais presentes na solução, principalmente quando a solução aquosa era sulfúrica. No estudo, a presença do ferro interferiu na extração do cobalto, assim como ocorreu neste estudo. A curva de extração do cobalto é deslocada para valores de $\mathrm{pH}$ inferiores quando o íon metálico está na presença do ferro em solução.

É sugerido pela literatura que o cobalto coextrai na solução orgânica na forma de complexos com o ferro [16][20], ou seja, na Equação 1 a extração forma o composto $(\mathrm{Co}, \mathrm{Fe}) \mathrm{R}_{\mathrm{n}}$.

\section{CONCLUSÃO}

O estudo da extração por solventes de uma solução sintética baseada no minério limonítico de níquel mostrou que até pH 2,0 não há extração dos metais magnésio, alumínio, manganês, níquel, cobre, cromo e zinco utilizando Cyanex 272 20\% em querosene. No entanto, entre $\mathrm{pH} 0,5$ e 2,0 há crescente extração do ferro para a solução orgânica, com é previsto pela seletividade teórica do extratante.

O cobalto foi coextraído com o ferro nos valores de $\mathrm{pH}$ estudados, contrariando a expectativa de extração desse metal que era previsto para valores de $\mathrm{pH}$ acima de 5,0 .

Em solução monoelementar de ferro, este metal se comporta de forma semelhante ao estudo realizado com solução multielementar quando ensaiado nas mesmas condições.

Já o cobalto tem comportamento diferenciado quando em solução monoelementar, sugerindo que a presença do ferro altera a curva de extração do cobalto para valores $\mathrm{pH}$ a partir de 0,5 . 


\section{Agradecimentos}

À Coordenação de Aperfeiçoamento de Pessoal de Nível Superior (CAPES) pelo suporte financeiro através da bolsa de mestrado.

Ao Conselho Nacional de Desenvolvimento Científico e Tecnológico (CNPq) pelo suporte financeiro através da bolsa de doutorado.

À Fundação de Amparo à Pesquisa do Estado de São Paulo (FAPESP) pelo suporte financeiro através do projeto de pesquisa 2012/51871-9.

\section{REFERÊNCIAS}

1 Georgiou D, Papangelakis VG. Sulphuric acid pressure leaching of a limonitic laterite: chemistry and kinetics. Hydrometallurgy, 1998; 49: 23-46.

2 Gupta CK. Chemical Metallurgy Principles and Practice. Weinheim: Wiley-VCH Verlag $\mathrm{GmbH} \&$ Co. KGaA; 2003.

3 Crundwell FK, Moats MS, Ramachandran V, Robinson TG, Davenport WG. Extractive Metallurgy of Nickel, Cobalt and Platinum Group Metals. Elsevier; 2011.

$4 \quad$ Kesler SE, Simon AC. Mineral Resources, Economics and the Environment. $2^{\mathrm{a}}$ ed. Cambridge University Press; 2015.

5 Guo X, Li D, Park KH, Tian Q, Wu Z. Leaching behavior of metals from a limonitic nickel laterite using a sulfation-roasting-leaching process, Hydrometallurgy, 2009; 99:144-150.

6 Ciminelli VST. Hidrometalurgia. In: Fernandes FRC, Matos GMM, Castilhos ZC, Luz AB (Eds.). Tendências tecnológicas Brasil 2015: geociências e tecnologia mineral. Rio de Janeiro: CETEM/MCT; 2007.

7 Vogel Al. Análise química quantitativa. 5a Edição. Filadélfia: LTC; 1992.

8 Morais CA, Albuquerque RO, Ladeira ACQ. Processos Físicos e Químicos Utilizados na Indústria Mineral. Cadernos Temáticos Química Nova Escola. 2014; 8: 9-17.

9 Habashi F. Principles of Extractive Metallurgy. Volume 2. Nova lorque: Gordon and Brach Science Publishers; 1970.

10 Kislik VS. Solvent Extraction: Classical and Novel Approaches. Elsevier; 2012.

11 Free M. Hydrometallurgy: fundamentals and applications. John Wiley \& Sons; 2013.

12 Wilson AM, Bailey PJ, Tasker PA, Turkington JR, Grant RA, Love JB. Solvent extraction: the coordination chemistry behind extractive metallurgy. Chemical Society Reviews. 2014; 43:123-134.

13 CYTEC. CYANEX 272 Extractant, Construction Materials. 2008.

14 Flett DS. Cobalt-Nickel Separation in Hydrometallurgy: a Review. Chemistry for Sustainable Development. 2004; 12: 81-91.

15 Flett DS. Solvent extraction in hydrometallurgy: the role of organophosphorus extractants. Jornal of Organometallic Chemistry. 2005; 690: 2426-2438.

16 Olivier MC, Dorfling C, Eksteen JJ. Evaluating a solvent extraction process route incorporating nickel preloading of Cyanex 272 for the removal of cobalt and iron from nickel sulphate solutions. Minerals Engineering. 2012; 27-28: 37-51.

17 Ritcey GM, Ashbrook AW. Solvent Extraction: principles and applications to process metallurgy - Parte1. Elsevier, 1984.

18 Chang Y, Zhai X, Li B, Fu Y. Removal of iron from acidic leach liquor of lateritic nickel ore by goethite precipitate. Hydrometallurgy. 2010; 101:84-87.

19 Hubicki Z, Hubicka H. Studies on the extraction process of nickel (II) sulphate purification using Cyanex 272. Hydrometallurgy. 1996; 40: 65-76.

20 Doyle FM, Pouillon D, Villegas EA. Solvent extraction of metals with carboxylic acids Coextraction of base metals with $\mathrm{Fe}(\mathrm{III})$ and characterization of selected carboxylate complexes. Hydrometallurgy. 1988; 19: 289-308. 